\title{
AS ASSEMBLEIAS DE PEIXES DOS ESTUÁRIOS DE CONCEIÇÃO DA BARRA E BARRA NOVA, ESPÍRITO SANTO.
}

\author{
M. HOSTIM-SILVA \\ A. C. LIMA $^{1}$ \\ J. DAMASCENO ${ }^{1}$ \\ T. SCIARRETTA ${ }^{1}$ \\ J. V. SILVA \\ R. L. BOT NETO ${ }^{2}$ \\ B. M. CARVALHO \\ H. L. SPACH ${ }^{2}$
}

Recebido em: 12/12/2012

Aceito em: 08/11/2013

\section{RESUMO}

Coletas mensais foram realizadas em quatro pontos amostrais de cada um dos estuários de Conceição da Barra e Barra Nova, utilizando-se para tal uma rede de arrasto de fundo com portas. Um total de 6243 indivíduos foi coletado pertencente a 55 taxa (28 famílias). Nos dois estuários as espécies mais abundantes foram Stellifer sp. e Diapterus rhombeus. Em ambos os estuários foram detectadas diferenças temporais na composição da ictiofauna, o mesmo tendo sido observado entre os pontos de coleta do estuário de Conceição da Barra.

Palavras chave: ictiofauna, variação espacial, variação temporal, Brasil.

\section{ABSTRACT}

Monthly samples were collected in four sampling points in each of the estuaries of Conceição da Barra and Barra Nova, using a bottom trawl with doors. A total of 6243 individuals were collected belonging to 55 taxa (28 families). The most abundant species in the estuaries were
Stellifer sp. Diapterus rhombeus. In both estuaries were detected temporal differences in the composition of the fish fauna, the same was observed between samples collected from the estuary of Conceição da Barra.

Key words: fish assemblage, spatial variation, temporal variation, Espírito Santo, Brazil

\section{INTRODUÇÃO}

Os estuários são áreas de transição entre rios e oceano, do tipo ecoclina, pois possuem comunidades heterogêneas que apresentam mudanças graduais em resposta ao gradiente ambiental (DE CAMPAS; NAIMAN, 2002). Apresentam grandes flutuações na salinidade, temperatura, oxigênio dissolvido, nutrientes, maré, fluxo de água doce, corrente marinha e turbidez (KUPSCHUS; TREMAIN, 2001). As mudanças nestes parâmetros físicos e químicos podem ser de curto prazo, sazonal e interanual, as quais exercem grande influência nas comunidades aquáticas (DAY et al., 1989), inclusive na comunidade íctica.

Os padrões espaciais e temporais nas comunidades estuarinas tem sido relacionados com o estresse fisiológico resultante de gradientes físicos e químicos (MAES et al., 1998), profundidade (HYNDES et al., 1999), distância da desembocadura, velocidade da corrente marinha (LONERAGAN et al. 1987; LONERAGAN et al., 1989), tolerância à salinidade (MARSHALL; ELLIOTT, 1998; WHITFIELD, 1999), temperatura (JAUREGUIZAR et al., 2003; JAUREGUIZAR et al., 2004) e turbidez (BLABER; BLABER, 1980; CYRUS; BLABER, 1992). Conjuntamente, exercem interferência na distribuição das espécies, fatores bióticos como as relações interespecíficas (efeito predador-presa) e interações (KENNISH, 1986).

1 Universidade Federal do Espírito Santo, Centro Universitário Norte do Espírito Santo, Departamento de Ciências Agrárias e Biológicas. mhostim@gmail.com; jdamascenobh@gmail.com; aclpesca@yahoo.com.br 2 Laboratório de Ecologia de Peixes, Centro de Estudos do Mar (CEM), Universidade Federal do Paraná (UFPR).bot@ufpr.br; bmaicarvalho@gmail.com; henry@ufpr.br 
HOSTIM-SILVA, M. et. al. As assembleias de peixes dos estuários de conceição da barra e barra nova, Espírito Santo.

Apesar das rápidas mudanças nas condições ambientais, os estuários funcionam como ambientes de criação para muitas espécies de peixes, em função da grande produtividade e suprimento de alimento (CARMOUZE, 1994); baixa transparência (PATTERSON; WHITFIELD, 2000) e baixa incidência de piscívoros (KENNISH, 1986).

Este trabalho tem por objetivo descrever composição, estrutura e as possíveis variações espaciais e temporais das assembleias de peixes que utilizam as áreas amostradas nos estuários de Conceição da Barra e Barra Nova (Espírito Santo), pois são escassos os estudos sobre os peixes em estuários do Espírito Santo (JOYEUX et al., 2004; REZENDE, 2005; MACIEIRA, 2005 ; CHAGAS et al., 2006; COSER et al., 2007). Assim, estudos sobre a fauna de peixes da região são de grande importância para o conhecimento básico das espécies, que em muitos casos podem estar desaparecendo ou sofrendo mudanças na sua biologia devido à ação antrópica.

\section{ÁREA DE ESTUDO}

A bacia do rio Cricaré localizada ao norte do Estado do Espírito Santo, abrange uma área aproximada de $13.500 \mathrm{~km}^{2}$. Formada pelos rios Cotaxé e Cricaré, ambos com suas nascentes situadas em Minas Gerais. A confluência desses rios ocorre no município de São Mateus, sendo chamado de rio Cricaré ou São Mateus. O rio Cricaré direciona-se para o norte e deságua no Oceano Atlântico, em Conceição da Barra, ES (SILVA et al., 2005). Afluente do rio Cricaré, o rio Mariricu, direciona-se para o sul, desaguando na comunidade de Barra Nova em São Mateus, formando o estuário de Barra Nova (Figura 1).

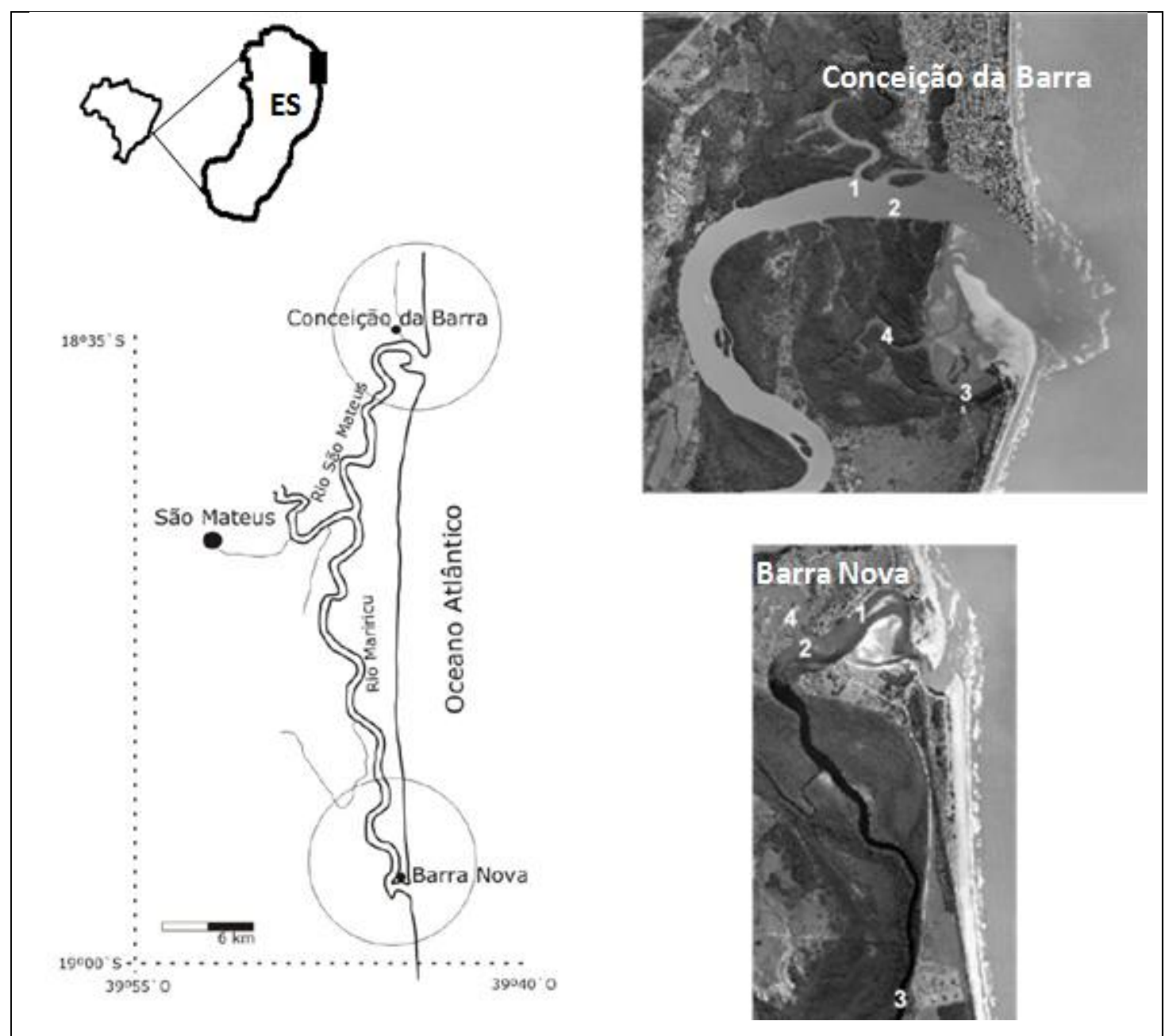

Figura 1 - Localização dos estuários do Rio Cricaré nos municípios de Conceição da Barra ao norte e São Mateus (Barra Nova) ao sul. Locais de amostragem no estuário de Conceição da Barra: 1 - Desembocadura do Rio Santana; 2 - Canal principal do Rio Cricaré; 3 - Bininho; 4 - Pitangueira. Locais de amostragem no estuário de Barra 
HOSTIM-SILVA, M. et. al. As assembleias de peixes dos estuários de conceição da barra e barra nova, Espírito Santo.

Nova: 1 - Cais para embarque e desembarque; 2 - Desembocadura do Rio Mariricu; 3 - Próximo a estação para armazenamento e transporte de petróleo (Transpetro); 4 - Rio Mariricu. Imagens de satélite disponibilizadas pelo Sistema Integrado de Bases Geoespaciais do Estado do Espírito Santo (http://www.geobases.es.gov.br/portal/index.php/navegadores.html).

O estuário do rio Cricaré em Conceição da Barra (39043'56.3"W / 18035'59.8"S) é caracterizado principalmente pela vegetação de manguezal composta por Rhizophora mangle (mangue vermelho) e Laguncularia racemosa (mangue branco) que ocupa $11 \mathrm{~km}^{2}$ (SILVA et al., 2005). O estuário de Barra Nova (39044'47.5"W / 18057"05.3"S), na confluência do rio Cricaré com o rio Mariricu até a foz predominam bosques de Avicennia schaueriana (mangue preto) e $A$. germinans (mangue preto) no alto curso, bosques mistos de $R$. mangle, $L$. racemosa e $A$. schaueriana no médio curso e $R$. mangle no baixo curso do rio. A foz do rio Barra Nova chega a fechar em determinadas épocas do ano(SILVA et al., 2005).

Os estuários do rio Cricaré apresentam marés semidiurnas. De acordo com os dados do Terminal de Barra do Riacho, município de Aracruz, ES (19050' S; 400.' W) no período estudado (Nov/2010 a Out/2011), a média das marés foi de $0,78 \mathrm{~m}$, com intervalo médio entre $-0,04$ e 1,51 m (Diretoria de Hidrografia e Navegação, Ministério da Marinha). O clima é classificado como tropical úmido. Considerando o período do desenvolvimento do trabalho, a temperatura média foi de $23,3^{\circ} \mathrm{C}$, com temperatura máxima de $34,5^{\circ} \mathrm{C}$ em fevereiro de $2011 \mathrm{e}$ mínima de $12,0^{\circ} \mathrm{C}$ em agosto de 2011. A região apresenta duas estações bem definidas, período quente entre novembro e abril, e um período com clima mais ameno entre maio e outubro (Figura 2).

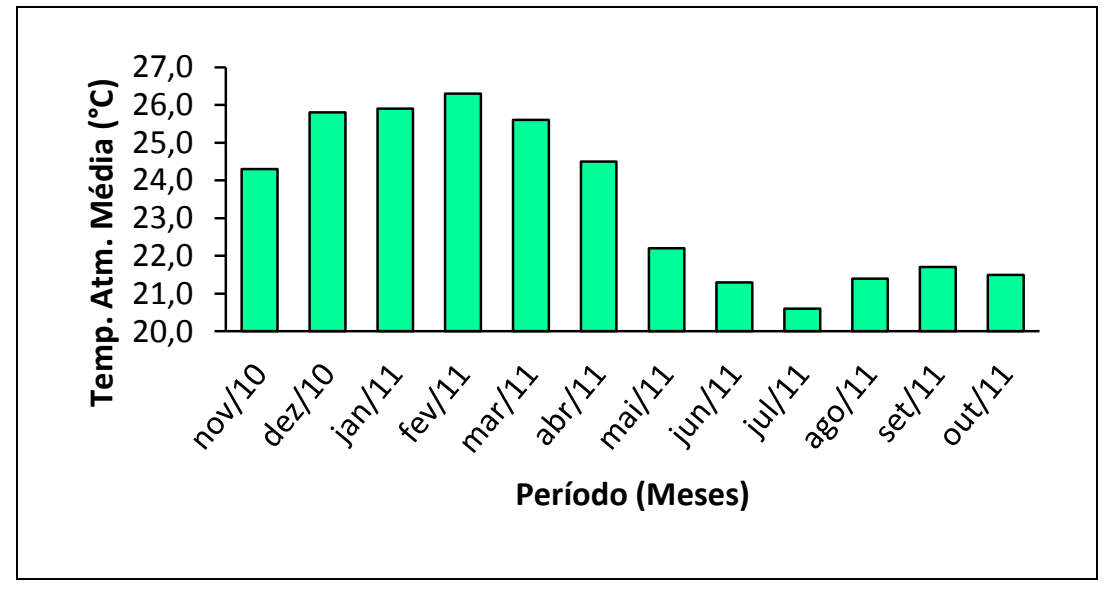

Figura 2 - Temperatura atmosférica média $\left({ }^{\circ} \mathrm{C}\right)$ nos estuários de Conceição da Barra e Barra nova entre novembro de 2010 e outubro de 2011.

A pluviosidade observada mensalmente no período, variou de 15,4 mm (Setembro/2011) a 239,8 mm (Abril/2011), sem estações bem definidas (Figura 3) (Dados meteorológicos médios, para o município de São Mateus-ES - LAT: 18,714S LON: 39,848W ALT: 39m), obtidos na estação meteorológica automática do INMET- disponibilizado pelo Instituto Capixaba de Pesquisa e Extensão Rural). 


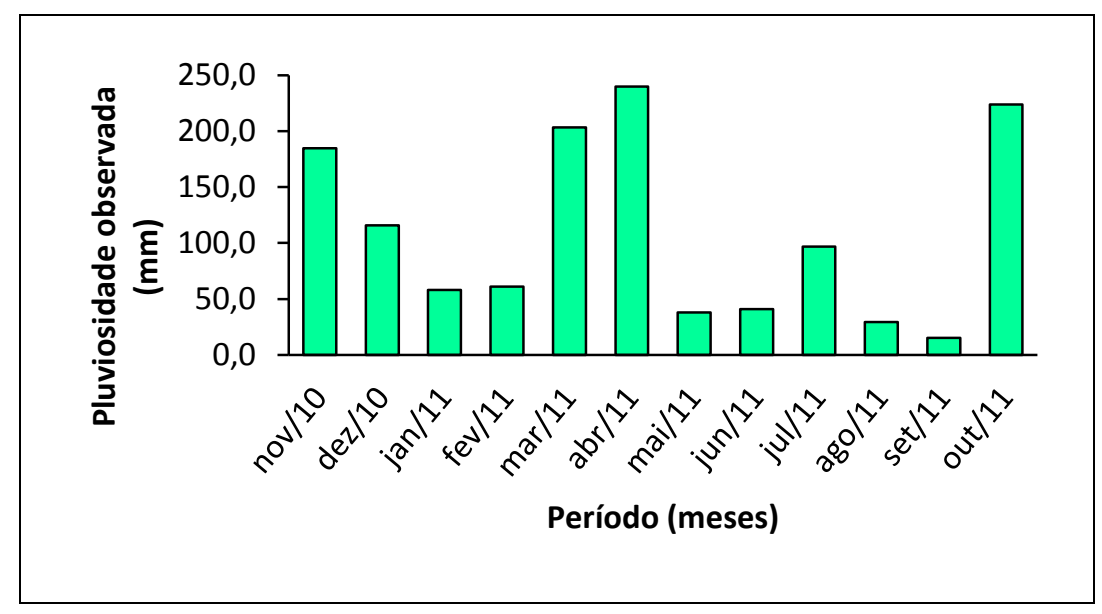

Figura 3 - Pluviosidade nos estuários de Conceição da Barra e Barra nova entre novembro de 2010 e outubro de 2011.

\section{MATERIAIS E METODOS}

\section{Coleta de dados}

As coletas foram realizadas em quatro pontos amostrais nos dois estuários do rio Cricaré: Conceição da Barra ao norte (Figura 1) e Barra Nova/São Mateus ao sul (Figura 1), entre novembro de 2010 e outubro de 2011.

As amostras foram obtidas mensalmente utilizando redes de arrasto de fundo com portas ( $2,80 \mathrm{~m}$ de abertura de boca por $3,90 \mathrm{~m}$ de comprimento e distância entre nós opostos de $3,9 \mathrm{~cm}$ no corpo e $2,2 \mathrm{~cm}$ no saco da rede), durante a maré alta, em cada uma das quatro áreas escolhidas foram realizados três arrastos de fundo (de cinco minutos cada) a uma velocidade média de $2,9 \mathrm{Km} / \mathrm{h}$.

Imediatamente após cada amostragem todo material coletado foi devidamente codificado, armazenado em sacos plásticos, acondicionados sob refrigeração e encaminhado ao Laboratório de Zoologia do Centro Universitário Norte do Espírito Santo para identificação e biometria. A identificação dos peixes foi feita ao menor nível taxonômico possível com o auxílio de literatura especializada (BARLETTA; CORRÊA, 1992; FIGUEIREDO; MENEZES, 1978, 1980 e 2000, MENEZES; FIGUEIREDO, 1980 e 1985). A seguir os indivíduos foram mensurados (comprimento total e comprimento padrão com precisão de $1,0 \mathrm{~mm}$ ) e pesados (peso total com precisão de $0,01 \mathrm{~g})$.

Diferenças entre as médias mensais e espaciais em cada estuário do número de espécies, número de indivíduos, diversidade de Shannon-Wiener $\left(\mathbf{H}^{\prime}=-(\mathbf{p i} * \mathbf{L n}(\mathbf{p i}))\right.$, onde $\mathbf{p i}=$ proporção da espécie $i$ na amostra total, e $\mathbf{s}=$ número de espécies, e o i variando de 1 a $\mathbf{s}$ ) e Equitabilidade de Pielou ( $\mathbf{J}=\mathbf{H}^{\prime}$ / H' Max) foram avaliadas através de análise de variância (ANOVA) unifatorial, com os pressupostos de normalidade e homogeneidade de variância sendo testados através dos testes de Kolmogorov-Smirnov e de Bartlett, respectivamente (SOKAL; ROHLF, 1995). Onde ocorreram diferenças significativas $(p<0,01$ e $p<0,05)$, aplicou-se o teste a posteriori de Tukey (SOKAL E ROHLF, 1995).

Para cada estuário em separado, foi aplicada a todas as espécies, o método de escalonamento multidimensional não linear (nMDS) (CLARKE; WARWICH, 1994), a partir da geração de uma matriz de similaridade através do coeficiente de Bray-Curtis, sem a transformação dos dados de freqüência absoluta, para avaliar se as assembleias apresentaramse diferentes entre os meses e pontos de coleta. Para a avaliação da significância das diferenças entre os grupos de meses e pontos de coleta visualizados no MDS, foi utilizada a análise de agrupamento Cluster e o teste Simprof, enquanto que as espécies que mais contribuíram com as dissimilaridades entre os grupos de meses e pontos de coleta de cada estuário foram identificadas através da análise SIMPER (CLARKE; WARWICH, 1994). 
HOSTIM-SILVA, M. et. al. As assembleias de peixes dos estuários de conceição da barra e barra nova, Espírito Santo.

\section{RESULTADOS}

Nesse estudo um total de 6243 indivíduos foram coletados nos estuários de Conceição da Barra (3159) e Barra Nova (3084), classificados em 55 taxa (28 famílias), 44 em Conceição da Barra e 46 em Barra Nova (tab. 1), com apenas três taxa de água doce (Oreochromis niloticus, Prochilodus sp. e Pseudauchenipterus nodosus). As famílias com maior número de espécies no estuário de Conceição da Barra foram Sciaenidae (7), Gerreidae (3), Engraulidae (3), Carangidae (3) e Haemulidae (3), já em Barra Nova a maior diversidade de espécies ocorreram em Sciaenidae (8), Gerreidae (6) e Carangidae (3).

As espécies mais abundantes nos dois estuários foram Stellifer sp. e Diapterus rhombeus representando $54 \%$ do número e $39 \%$ do peso total das espécies capturadas. Também tiveram uma contribuição significativa para a abundância e biomassa nos dois estuários as espécies Achirus declives e Sphoeroides testudineus, com grande contribuição só para a biomassa de Cathorops spixii e Genidens genidens em Conceição da Barra e de Bairdiella ronchus, Centropomus paralellus, Centropomus undecimalis, Gobioides broussonnetii e Micropogonias furnieri na Barra Nova (tab.1). Nas áreas estudadas, 30 espécies de peixes contribuíram cada uma com menos de $1 \%$ da captura total de cada área.

Só estiveram presentes nas amostras de Conceição da Barra as espécies Anchovia clupeoides, Anchoviella brevirostris, Caranx crysus, Caranx latus, Cynoscion leiarchus, Gymnothorax funebris, Odontognathus mucronatus, Pomadasys ramosus, Prochilodus sp. e Trichiurus lepturus (tab.1). Foram exclusivas da Barra do Sul as espécies Epinephelus itajara, Eugerres brasilianus, Gobioides broussonnetii, Larimus breviceps, Mugil liza, Ophichthus parilis, Ophioscion punctatissimus, Oreochromis niloticus, Prionotus punctatus e Synodus foetens.

Em média foram maiores em Conceição da Barra os exemplares de Paralichthys brasiliensis, Centropomus undecimalis, Micropogonias furnieri e Genidens genidens, com as espécies Mugil liza, Gobioides broussonnetii, Mugil curema, Paralichthys brasiliensis e Centropomus undecimalis apresentando os maiores tamanhos médios na Barra Nova (tab.1). Maiores amplitudes de tamanhos foram observadas em Paralichthys brasilianus, Genidens genidens e Cysnoscion acoupa no estuário de Conceição da Barra, já na região de Barra Nova a amplitude de tamanho foi maior em Cynoscion acoupa, Centropomus paralellus e Centropomus undecimalis. 
M. HOSTIM-SILVA et al. As assembleias de peixes dos estuários de conceição da barra e barra nova, espírito santo.

Tabela 1 - Lista de espécies capturadas nos estuários de Conceição da Barra e Barra Nova entre os meses de novembro de 2010 e outubro de 2011. N, número de indivíduos; CT, comprimento total; DP, desvio padrão.

\begin{tabular}{|c|c|c|c|c|c|c|}
\hline & Conceição da Barra & Barra Nova & & & & \\
\hline ESPÉCIE & $\mathrm{N}$ & $\mathrm{CT}_{\text {médio }}($ Mín-Máx $) \pm \mathrm{DP}(\mathrm{mm})$ & Biomassa (g) & $\mathrm{N}$ & $\mathrm{CT}_{\text {médio }}($ Mín-Máx $) \pm \mathrm{DP}(\mathrm{mm})$ & Peso total $(\mathrm{g})$ \\
\hline Achirus declivis & 205 & $95(39-180) \pm 28,8$ & 4785 & 126 & $72(43-150) \pm 15$ & 1034 \\
\hline Achirus lineatus & 77 & $86(42-114) \pm 14,7$ & 1042 & 36 & $58(40-75) \pm 8$ & 135 \\
\hline Anchovia clupeoides & 1 & 103 & 6 & - & - & - \\
\hline Anchoviella brevirostris & 1 & 87 & 2 & - & - & - \\
\hline Archosargus probatocephalus & 1 & 97 & 20 & 38 & $121(82-186) \pm 19$ & 1464 \\
\hline Bairdiella ronchus & 26 & $117(77-186) \pm 29,8$ & 655 & 200 & $163(60-210) \pm 27$ & 11862 \\
\hline Caranx crysos & 6 & $77(56-104) \pm 21$ & 43 & - & - & - \\
\hline Caranx latus & 24 & $73(34-115) \pm 22,7$ & 180 & - & - & - \\
\hline Cathorops spixii & 69 & $176(122-250) \pm 27,4$ & 4217 & 18 & $204(174-224) \pm 16$ & 1410 \\
\hline Centropomus parallelus & 15 & $185(135-269) \pm 36$ & 911 & 100 & $185(99-419) \pm 47$ & 6978 \\
\hline Centropomus undecimalis & 12 & $237(131-296) \pm 52,5$ & 1370 & 34 & $254(167-417) \pm 58$ & 4823 \\
\hline Cetengraulis edentulus & 63 & $142(96-170) \pm 15,9$ & 1677 & 57 & $144(79-194) \pm 21$ & 1446 \\
\hline Chaetodipterus faber & 5 & $76(47-145) \pm 40,2$ & 165 & 8 & $78(45-140) \pm 31$ & 170 \\
\hline Chloroscombrus chrysurus & 16 & $108(52-139) \pm 21$ & 194 & 1 & 115 & 12 \\
\hline Citharichthys spilopterus & 99 & $99(55-165) \pm 21,7$ & 920 & 35 & $108(59-180) \pm 26$ & 446 \\
\hline Cyclichthys spinosus & 64 & $74(60-113) \pm 9,4$ & 2139 & 1 & 62 & 28 \\
\hline Cynoscion acoupa & 57 & $133(51-287) \pm 55,6$ & 1741 & 38 & $136(36-414) \pm 98$ & 2648 \\
\hline Cynoscion leiarchus & 13 & $127(52-183) \pm 42,8$ & 556 & - & - & - \\
\hline Diapterus auratus & 3 & $89(83-94) \pm 5,7$ & 30 & 27 & $127(98-282) \pm 40$ & 941 \\
\hline Diapterus rhombeus & 813 & $83(10-188) \pm 21,1$ & 8186 & 541 & $118(50-192) \pm 30$ & 15941 \\
\hline Epinephelus itajara & - & - & - & 1 & 169 & 71 \\
\hline Eucinostomus melanopterus & 2 & $145(132-158) \pm 18,4$ & 72 & 4 & $115(109-128) \pm 9$ & 72 \\
\hline Eugerres brasilianus & - & - & - & 1 & 174 & 61 \\
\hline Genidens genidens & 148 & $192(20-274) \pm 37,9$ & 9158 & 1 & 235 & 99 \\
\hline
\end{tabular}


M. HOSTIM-SILVA et al. As assembleias de peixes dos estuários de conceição da barra e barra nova, espírito santo.

Tabela 1- Lista de espécies capturadas nos estuários de Conceição da Barra e Barra Nova entre os meses de novembro de 2010 e outubro de 2011. N, número de indivíduos; CT, comprimento total; DP, desvio padrão.

(continuação)

\begin{tabular}{|c|c|c|c|c|c|c|}
\hline & \multicolumn{3}{|c|}{ Conceição da Barra } & \multicolumn{3}{|c|}{ Barra Nova } \\
\hline Espécie & $\mathrm{N}$ & $\mathrm{CT}_{\text {médio }}($ Mín-Máx) $\pm \mathrm{DP}(\mathrm{mm})$ & Biomassa (g) & $\mathrm{N}$ & $\begin{array}{c}\mathrm{CT}_{\text {médio }}(\text { Mín-Máx }) \pm \mathrm{DP} \\
(\mathrm{mm})\end{array}$ & $\begin{array}{l}\text { Peso total } \\
\text { (g) }\end{array}$ \\
\hline Genidens genidens & 148 & $192(20-274) \pm 37,9$ & 9158 & 1 & 235 & 99 \\
\hline Genyatremus luteus & 1 & 251 & 338 & 6 & $84(75-98) \pm 9$ & 75 \\
\hline Gobioides broussonnetii & - & - & - & 75 & $353(244-488) \pm 60$ & 5312 \\
\hline Gobionellus oceanicus & 2 & $122(114-131) \pm 12$ & 21 & 8 & $199(160-259) \pm 35$ & 108 \\
\hline Gymnothorax funebris & 1 & 793 & 1272 & - & - & - \\
\hline Lagocephalus laevigatus & 22 & $83(62-175) \pm 22,8$ & 401 & 19 & $110(59-277) \pm 56$ & 932 \\
\hline Larimus breviceps & - & - & - & 4 & $62(59-65) \pm 3$ & 9 \\
\hline Lutjanus jocu & 12 & $160(107-208) \pm 35,7$ & 954 & 10 & $171(139-207) \pm 24$ & 756 \\
\hline Micropogonias furnieri & 18 & $149(92-265) \pm 50,4$ & 824 & 180 & $131(71-218) \pm 31$ & 4471 \\
\hline Mugil curema & 16 & $205(124-312) \pm 48,5$ & 1586 & 4 & $299(229-360) \pm 55$ & 1014 \\
\hline Mugil liza & - & - & - & 3 & $445(311-533) \pm 118$ & 2540 \\
\hline Odontognathus mucronatus & 2 & $121(112-130) \pm 12,7$ & 13 & - & - & - \\
\hline Ophichthus parilis & - & - & - & 1 & 186 & 2 \\
\hline Ophioscion punctatissimus & - & - & - & 1 & 135 & 32 \\
\hline Oreochromis niloticus & - & - & - & 1 & 148 & 54 \\
\hline Paralichthys brasiliensis & 6 & $283(132-455) \pm 122,3$ & 2254 & 3 & $290(246-376) \pm 75$ & 975 \\
\hline Polydactylus oligodon & 30 & $157(112-252) \pm 26,8$ & 1232 & 28 & $174(116-230) \pm 29$ & 1536 \\
\hline Polydactylus virginicus & 3 & $147(136-163) \pm 14,2$ & 94 & 7 & $189(155-221) \pm 26$ & 471 \\
\hline Pomadasys corvinaeformis & 1 & 170,0 & 73 & 3 & $144(130-161) \pm 16$ & 139 \\
\hline Pomadasys ramosus & 1 & 384 & 680 & - & - & - \\
\hline Prionotus punctatus & - & - & - & 2 & $116(107-124) \pm 12$ & 37 \\
\hline Prochilodus sp. & 2 & $89(85-94) \pm 6,4$ & 16 & - & - & - \\
\hline
\end{tabular}


M. HOSTIM-SILVA et al. As assembleias de peixes dos estuários de conceição da barra e barra nova, espírito santo.

\begin{tabular}{|c|c|c|c|c|c|c|c|c|}
\hline Pseudauchenipterus nodosus & & 57 & $101(10-131) \pm 17,6$ & & 759 & 8 & $111(104-130) \pm 8$ & 121 \\
\hline Rypticus randalli & & 21 & $139(105-162) \pm 17,6$ & & 838 & 44 & $137(103-172) \pm 18$ & 1734 \\
\hline Selene vomer & & 13 & $86(42-163) \pm 34,4$ & & 168 & 8 & $70(52-125) \pm 25$ & 94 \\
\hline Sphoeroides testudineus & & 256 & $154(18-238) \pm 38,3$ & & 25243 & 203 & $130(50-231) \pm 30$ & 10904 \\
\hline Stellifer rastrifer & & 87 & $92(52-163) \pm 23,8$ & & 986 & 1 & 126 & 23 \\
\hline Stellifer sp. & & 844 & $132(61-179) \pm 22,9$ & & 24922 & 1168 & $139(62-189) \pm 21$ & 37028 \\
\hline Stellifer stellifer & & 7 & $133(112-155) \pm 13,1$ & & 219 & 2 & $111(109-112) \pm 2$ & 27 \\
\hline Symphurus tessellatus & & 42 & $154(95-195) \pm 28,6$ & & 1397 & 19 & $126(117-150) \pm 10$ & 264 \\
\hline Synodus foetens & - & & - & - & & 3 & $167(120-250) \pm 72$ & 135 \\
\hline Trichiurus lepturus & 1 & & 27 & 13 & & - & - & - \\
\hline Total & 3159 & & $119(10-793) \pm 45,9$ & 102327 & & 3084 & $140(36-533) \pm 54$ & 118474 \\
\hline
\end{tabular}


M. HOSTIM-SILVA et al. As assembléias de peixes dos estuários de conceição da barra e barra nova, espírito santo.

\section{Variação temporal}

Estiveram presentes em todos os meses de coleta Diapterus rhombeus e Stellifer sp. em ambos os estuários e Micropogonias furnieri e Sphoeroides testudineus somente na Barra Nova. Também apareceram na maioria dos meses de coleta as espécies Achirus declives, Achirus lineatus, Cetengraulis edentulus, Citharicthys spilopterus e Sphoeroides testudineus em Conceição da Barra e Archosargus probatocephalus, Centropomus paralellus, Centropomus undecimalis, Citharichthys spilopterus, Cynoscion acoupa e Rypticus randalli na Barra Nova. Em apenas um mês de coleta 10 e 15 espécies foram registradas nos estuários de Conceição da Barra e Barra Nova, respectivamente.

No estuário de Conceição da Barra não foi observada nenhuma diferença significativa entre as médias mensais do número de espécies (Fig. 4A), número de indivíduos (Fig. 4B), diversidade de Shannon-Wiener (Fig. 4C) e equitabilidade de Pielou (Fig. 4D). Entre os meses de coleta a diversidade média de Shannon-Wiener não diferiu na Barra Nova (Fig. 5C). Nesse estuário a média do número de espécies capturadas em cada mês de coleta foi significativamente menor em março em relação aos demais meses do ano, com exceção de novembro e fevereiro (Fig. 5A). Também foram diferentes as médias mensais do número de indivíduos, com maiores capturas em janeiro, abril e maio em comparação a março, agosto e novembro (Fig. 5B). Na Barra Nova ocorreram médias significativamente menores de equitabilidade em abril e maio quando comparadas com outubro, novembro e dezembro (Fig. 5D).
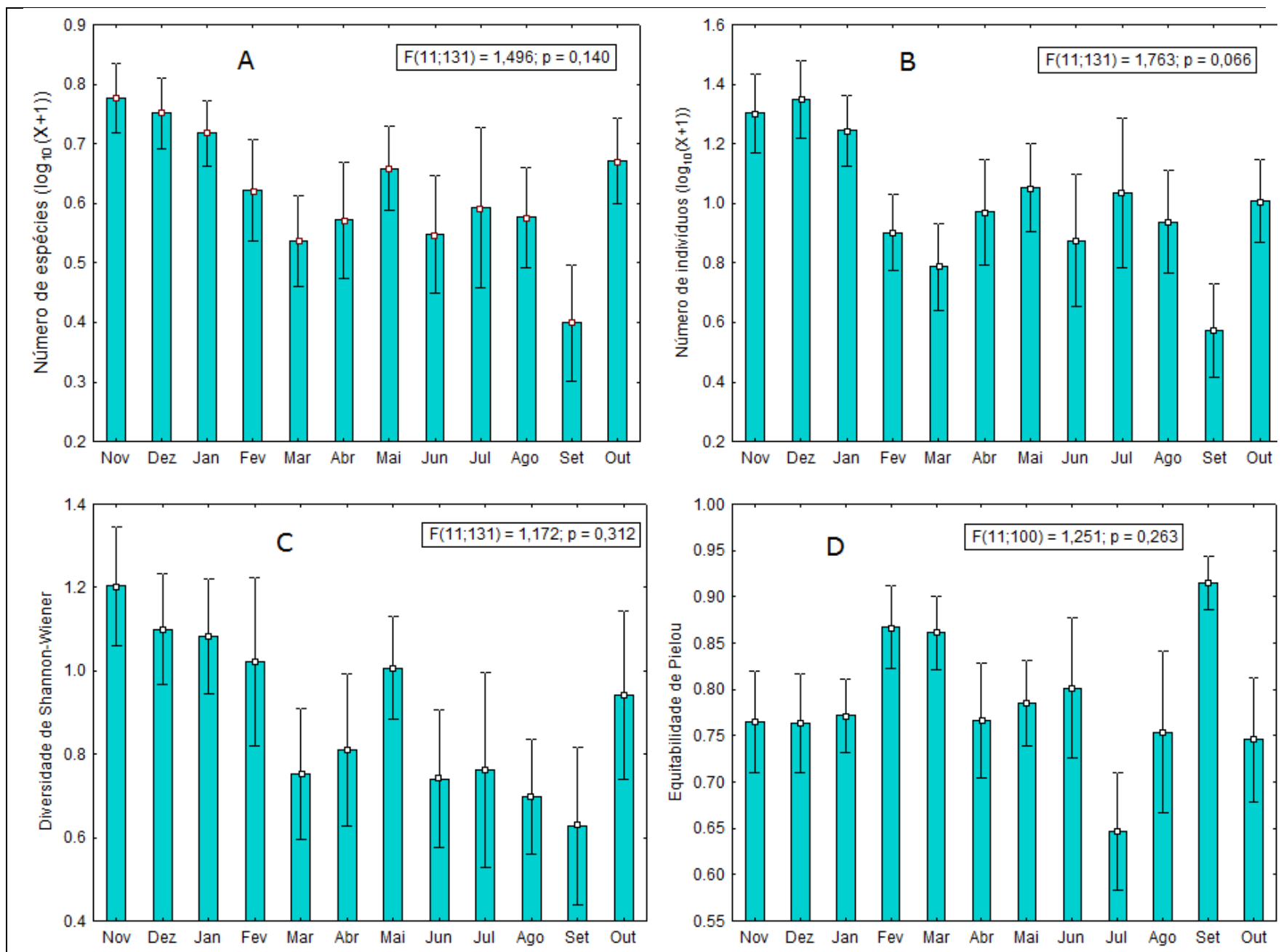

Figura 4 - Resultado da análise de variância avaliando as diferenças entre as médias mensais do número de espécies (A), número de indivíduos (B), diversidade de Shannon- 
M. HOSTIM-SILVA et al. As assembleias de peixes dos estuários de conceição da barra e barra nova, espírito santo.

Wiener (C) e Equitabilidade de Pielou (D) em Conceição da Barra, Espírito Santo (

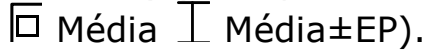
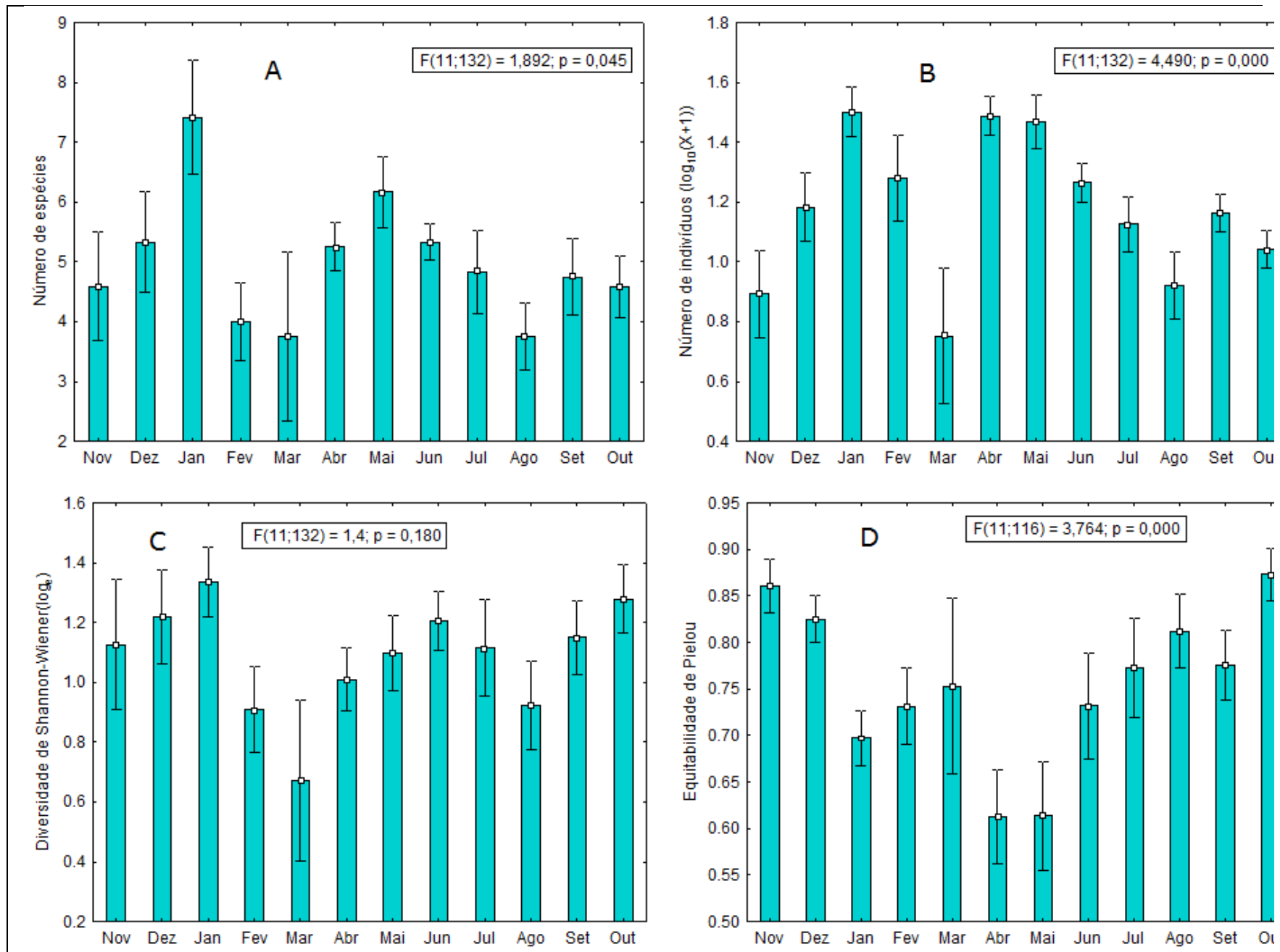

Figura 5 - Resultado da análise de variância avaliando as diferenças entre as médias mensais do número de espécies $(A)$, número de indivíduos $(B)$, diversidade de ShannonWiener (C) e Equitabilidade de Pielou (D) na Barra Nova, Espírito Santo (匡 Média I MédiatEP).

Com base nos dados de todas as espécies amostradas no estuário de Conceição da Barra, as análises de agrupamento (Cluster) e ordenamento (MDS) (Fig. 6), mostram a formação de dois grupos de meses. O grupo A formado pelos meses de setembro, outubro, novembro, dezembro, janeiro, fevereiro, março, abril e maio e o grupo B contendo os meses de junho, julho e agosto. Contribuiu mais para a similaridade média interna do grupo $A$ a maior abundância média de Stellifer sp., Achirus declives, Diapterus rhombeus, Cathorops spixii e Achirus lineatus. Já as relativas altas capturas de Diapterus rhombeus, Stellifer sp., Citharichthys spilopterus resultaram no agrupamento B. Os dois grupos separam-se pela alta captura de Achirus declives, Sphoeroides testudineus, Achirus lineatus e Cathorops spixii no grupo A e de Diapterus rhombeus, Citarichthys spilopterus, Lagocephalus laevigatus, Polidactylus oligodon, Caranx latus e Bairdiella ronchus no grupo B. 
M. HOSTIM-SILVA et al. As assembleias de peixes dos estuários de conceição da barra e barra nova, espírito santo.

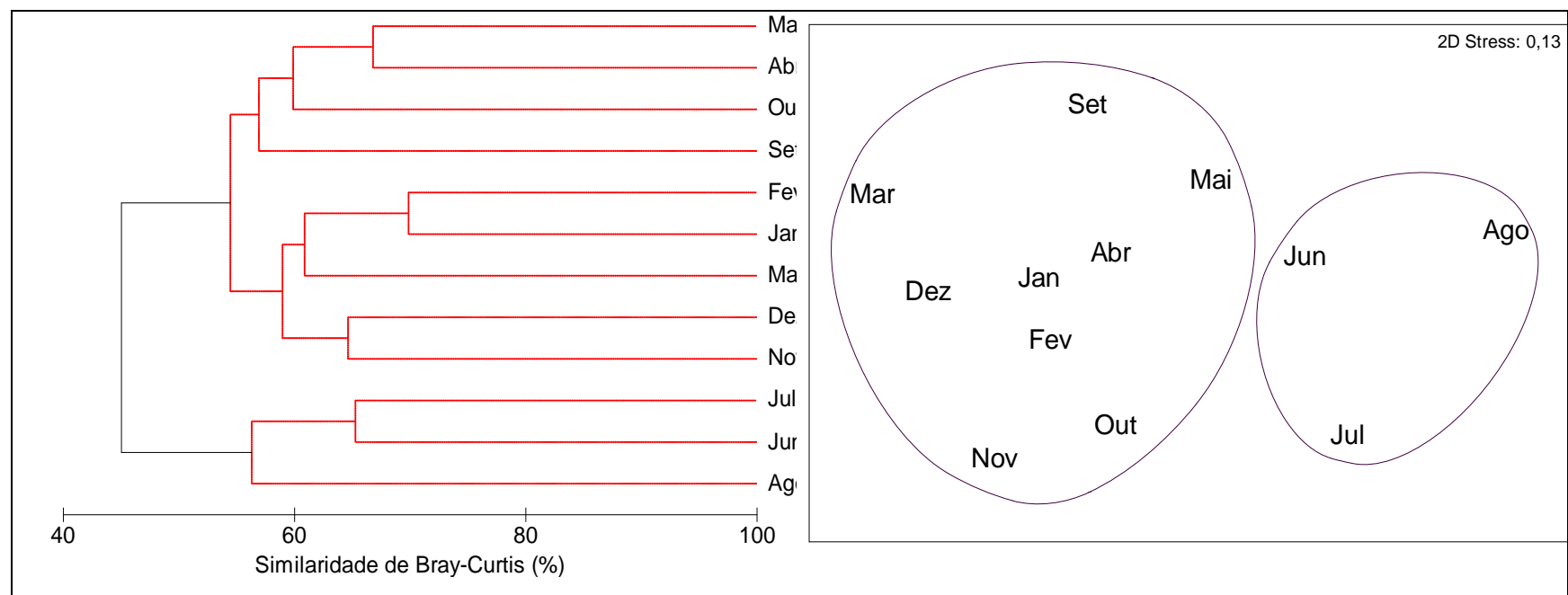

Figura 6 - Cluster e MDS utilizando como atributos a ocorrência e a abundância mensal das espécies capturadas em Conceição da Barra, Espírito Santo.

A utilização de todas as espécies capturadas no estuário da Barra Nova para compor a análise de agrupamento através do cluster hierárquico e o escalonamento multidimensional não métrico (MDS) revelou a formação de dois grupos principais de meses (Fig.7). Um primeiro grupo (A) é formado pelos meses de junho, julho, agosto, setembro, outubro, novembro e dezembro, quando foram predominantes em ordem de abundancia média, as espécies Diapterus rhombeus, Stellifer sp., Micropogonias furnieri, Sphoeroides testudineus e Centropomus parallelus. Um segundo grupo (B), este formado pelos meses de janeiro, fevereiro, março, abril e maio, que esteve representado em maior abundância pelas espécies Stellifer sp., Diapterus rhombeus, Sphoeroides testudineus, Bairdiella ronchus, Micropogonias furnieri e Achirus lineatus. A dissimilaridade entre os dois grupos deve-se principalmente as capturas em média maiores de Cetengraulis edentulus e Lagocephalus laevigatus no grupo A e de Achirus declives, Bairdiella ronchus, Achirus lineatus e Gobioides broussonnetii no grupo B.

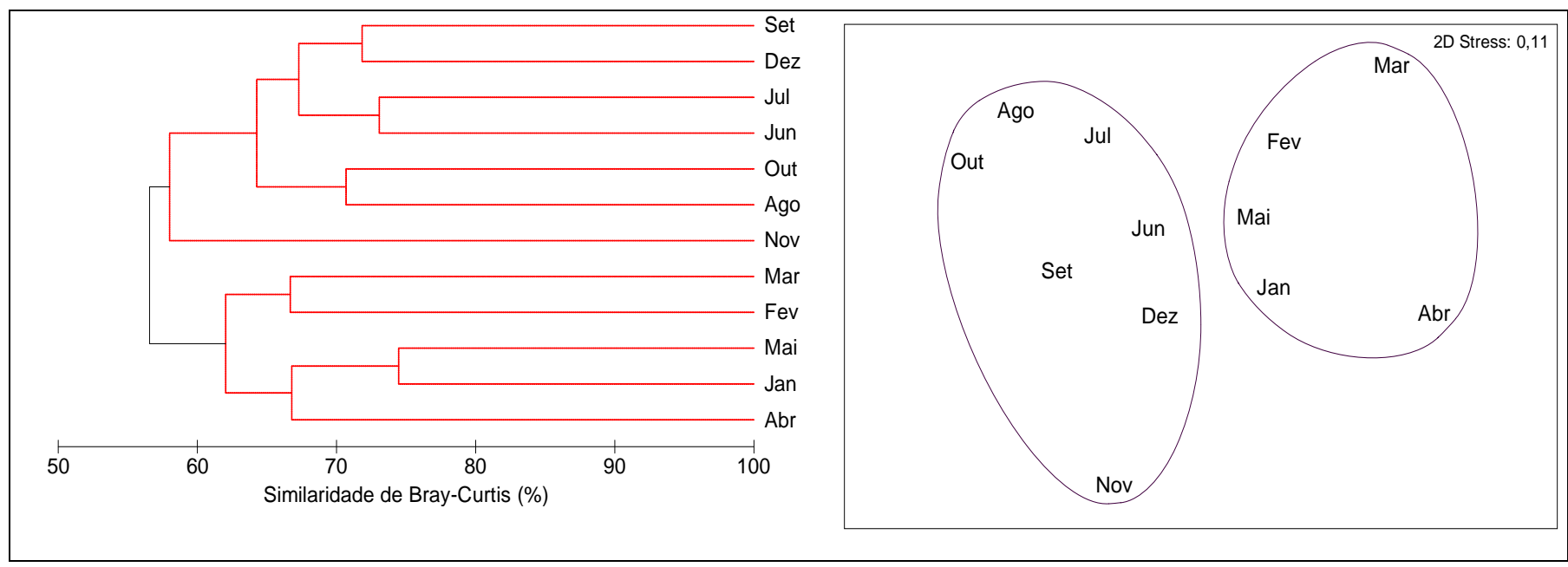

Figura 7 - Cluster e MDS utilizando como atributos a ocorrência e a abundância mensal das espécies capturadas na Barra Nova, Espírito Santo.

\section{Variação espacial}

Estiveram presentes em todos os pontos de coleta de Conceição da Barra as espécies Achirus lineatus, Cathorops spixii, Citharichthys spilopterus, Cyclichthys spinosus, Diapterus rhombeus, Micropogonias furnieri, Stellifer rastrifer e Stellifer sp., por outro lado, só apareceram em um ponto de coleta desse estuário as espécies Anchovia cluepoides, Anchoviella brevirostris, Archosargus probatocephalus, Caranx latus, Diapterus auratus, Eucinostomus melanopterus, Genyatremus luteus, Gobionellus oceanicus, Gymnothorax funebris, Lutjanus 
M. HOSTIM-SILVA et al. As assembleias de peixes dos estuários de conceição da barra e barra nova, espírito santo.

jocu, Odontognathus mucronatus, Pomadasys corvinaeformis, Pomadasys ramosus, Prochilodus sp. e Trichiurus lepturus.

No estuário de Barra Nova, a presença em todos os pontos de amostragem foi de Achirus declivis, Achirus lineatus, Archosargus probatocephalus, Bairdiella ronchus, Centropomus paralellus, Centropomus undecimalis, Cetengraulis edentulus, Citharichthys spilopterus, Cynoscion acoupa, Diapterus auratus, Diapterus rhombeus, Lagocephalus laevigatus, Lutajanus jocu, Micropogonias furnieri, Polydactylus oligodon, Rypticus randalli, Selene vomer, Sphoeroides testudineus e Stellifer sp.. Foram exclusivas de apenas um ponto de coleta Chloroscombrus chrysurus, Cyclichthys spinosus, Epinephelus itajara, Eugerres brasilianus, Genidens genidens, Gobioides broussonnetii, Gobionellus oceanicus, Larimus breviceps, Ophichthus parilis, Ophioscion punctatissimus, Orechromis niloticus, Paralichthys brasilianus, Polydactylus virginicus, Prionotus punctatus, Stellifer rastrifer, Stellifer stellifer e Synodus foetens.

A análise de variância e o teste de Tukey revelaram diferenças significativas no número médio de espécies entre os quatro pontos amostrais do estuário de Conceição da Barra (Fig. $8 \mathrm{~A}$ ), com médias maiores e diferentes entre si no Bininho e Pitangueira, e menores mais sem diferença significativa entre as médias da foz do rio Santana e do canal principal do rio Cricaré. Em média o número de indivíduos foram significativamente maiores e similares no Bininho e Pitangueira, com as áreas da foz do rio Santana e do canal principal do rio Cricaré apresentando médias menores e não diferentes entre si (Fig.8B).

As áreas de coleta no canal principal do rio Cricaré e na foz do rio Santana apresentaram médias de diversidade não diferentes entre si, mas menores quando comparadas com o Bininho e Pitangueira, essas últimas também similares entre si (Fig. 8C). Apesar do valor de $p=0,049$, o teste a posteriori de Tukey não apontou diferenças estatísticas entre as equitabilidades médias dos pontos amostrais no estuário de Conceição da Barra (Fig. 8D). 
M. HOSTIM-SILVA et al. As assembleias de peixes dos estuários de conceição da barra e barra nova, espírito santo.

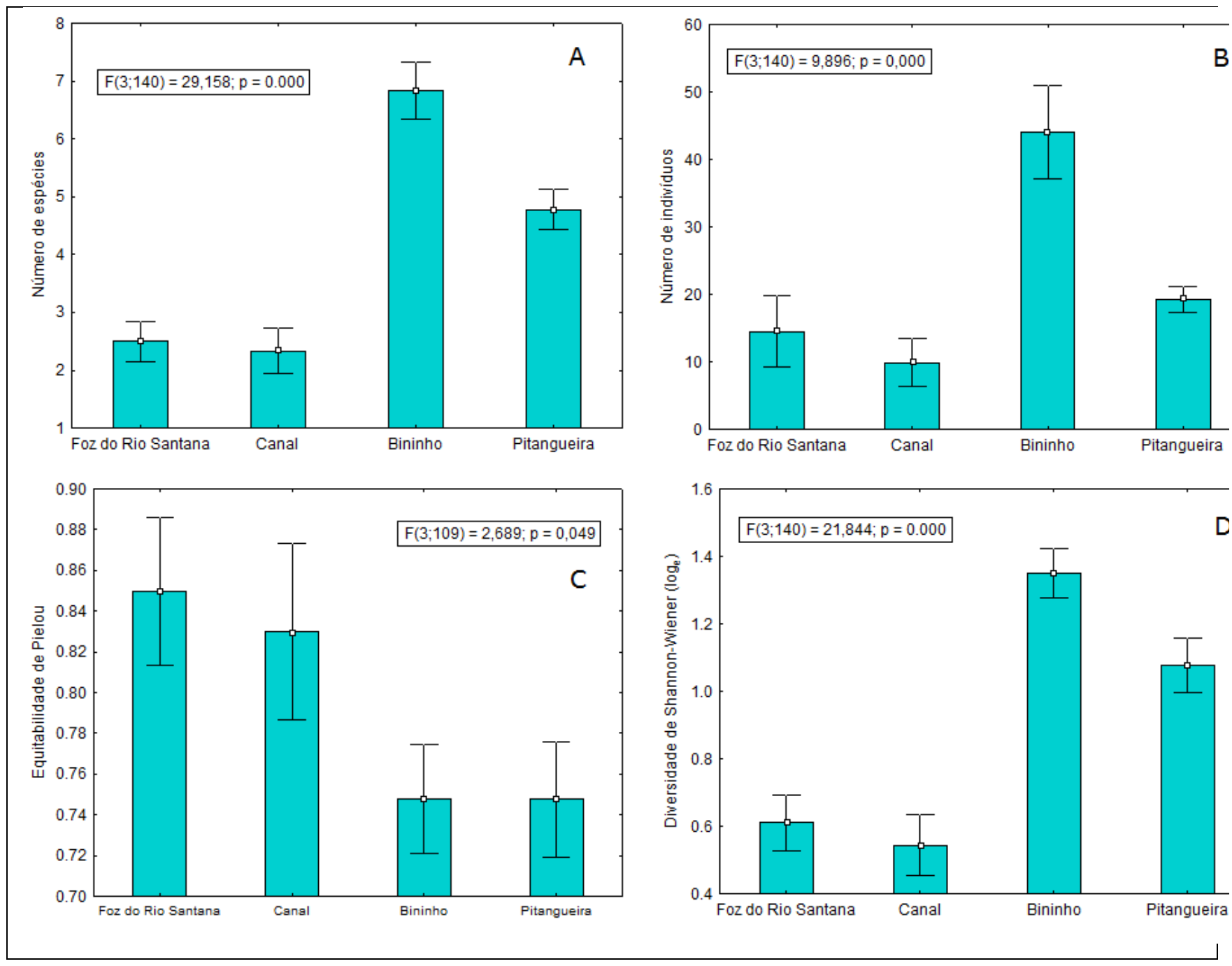

Figura 8 - Resultado da análise de variância avaliando as diferenças entre as médias mensais do número de espécies $(A)$, número de indivíduos (B), diversidade de ShannonWiener (C) e Equitabilidade de Pielou (D) em Conceição da Barra, Espírito Santo.

Analisando a média do número de espécies por ponto de coleta do estuário de Barra Nova, observou-se diferença significativa, com maior média no Cais em relação aos demais, esses com médias não diferentes entre si (Fig. 9A). Já para o número de indivíduos, a análise de variância entre as médias não revelou diferenças significativas entre os pontos amostrais (Fig.9B).

Em termos de diversidade, foi possível observar uma média significativamente maior no Cais em relação aos demais locais de coleta, uma diversidade média maior no rio Mariricu em comparação a localidade Transpetro e diversidades não diferenciando entre a foz e parte mais interna do rio Mariricu e entre a foz do rio Mariricu e a Transpetro (Fig. 9C). Após a aplicação da análise de variância aos dados de equitabilidade, verificou-se valores médios de equitabilidade significativamente maiores no Cais e rio Mariricu em relação a Transpetro, não diferindo entre as demais áreas de coleta (Fig. 9D). 
M. HOSTIM-SILVA et al. As assembleias de peixes dos estuários de conceição da barra e barra nova, espírito santo.
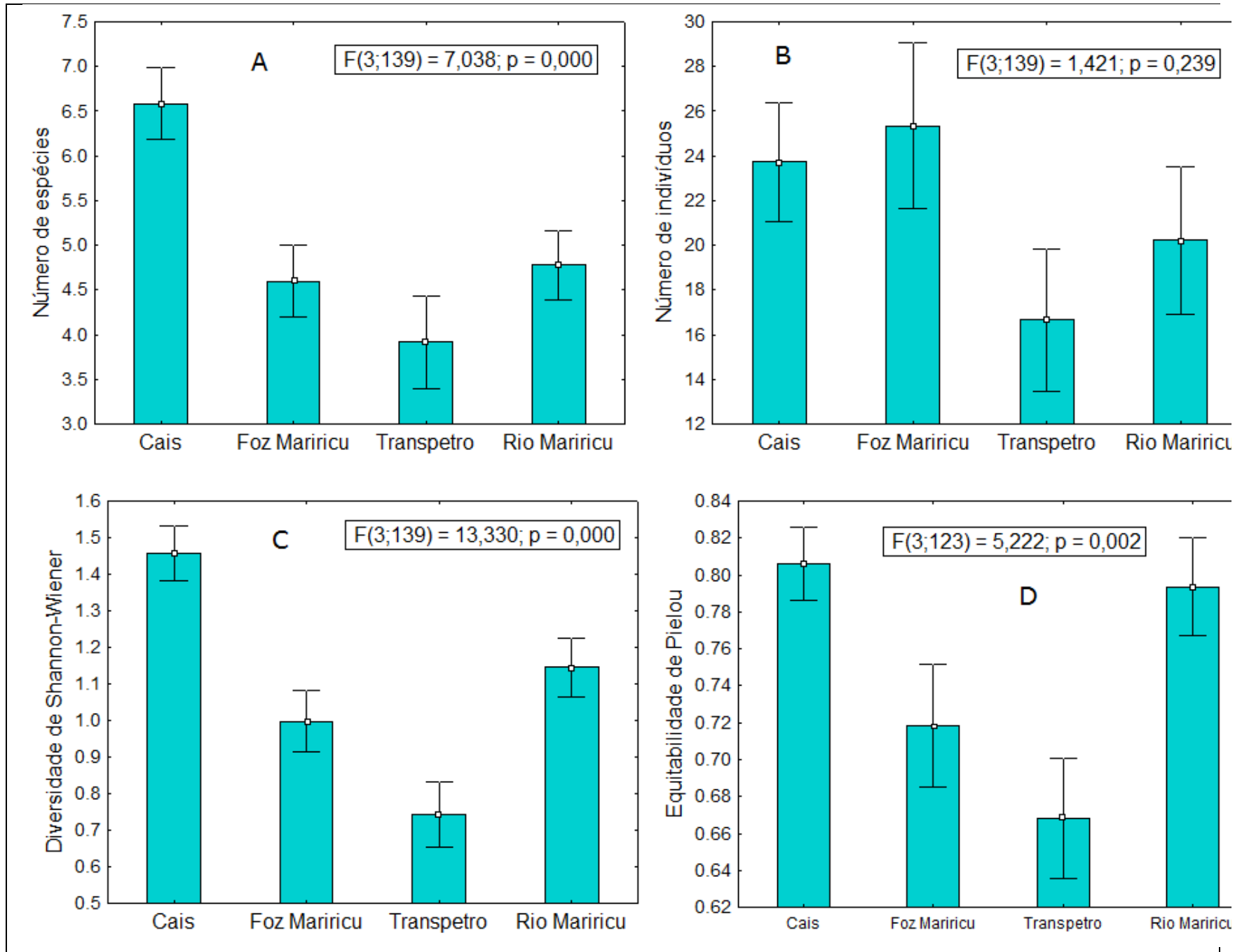

Figura 9 - Resultado da análise de variância avaliando as diferenças entre as médias mensais do número de espécies $(A)$, número de indivíduos (B), diversidade de ShannonWiener (C) e Equitabilidade de Pielou (D) na Barra Nova, Espírito Santo.

As análises de cluster e MDS com base na abundância de todas as espécies coletadas mostraram mudança na estrutura das assembleias de peixes entre as áreas de coleta no estuário de Conceição da Barra (Fig. 10). As assembleias amostradas na Pitangueira e Bininho, áreas fora do canal principal do rio Cricaré, são semelhantes entre si principalmente pelas abundancias médias de Stellifer sp., Diapterus rhombeus, Sphoeroides testudineus, Achirus declives, Citharichthys spilopterus e Cynoscion acoupa. A semelhança ictiofaunistica também foi observada entre a foz do rio Santana e o canal principal do rio Cricaré, contribuindo mais para isto a abundância média de Stellifer sp., Genidens genidens, Achirus lineatus, Pseudauchenipterus nodosus, Symphurus tesselatus e Stellifer rastrifer. Os dois grupos definidos nas análises de agrupamento e ordenamento, possuem assembleias relativamente diferentes, em função principalmente da maior abundancia média de Genidens genidens e Pseudauchenipterus nodosus nas áreas do canal principal do rio Cricaré e foz do rio Santana, e das espécies Achirus declives, Diapterus rhombeus, Cynoscion acoupa e Sphoeroides testudineus no Bininho e Pitangueira. 
M. HOSTIM-SILVA et al. As assembleias de peixes dos estuários de conceição da barra e barra nova, espírito santo.

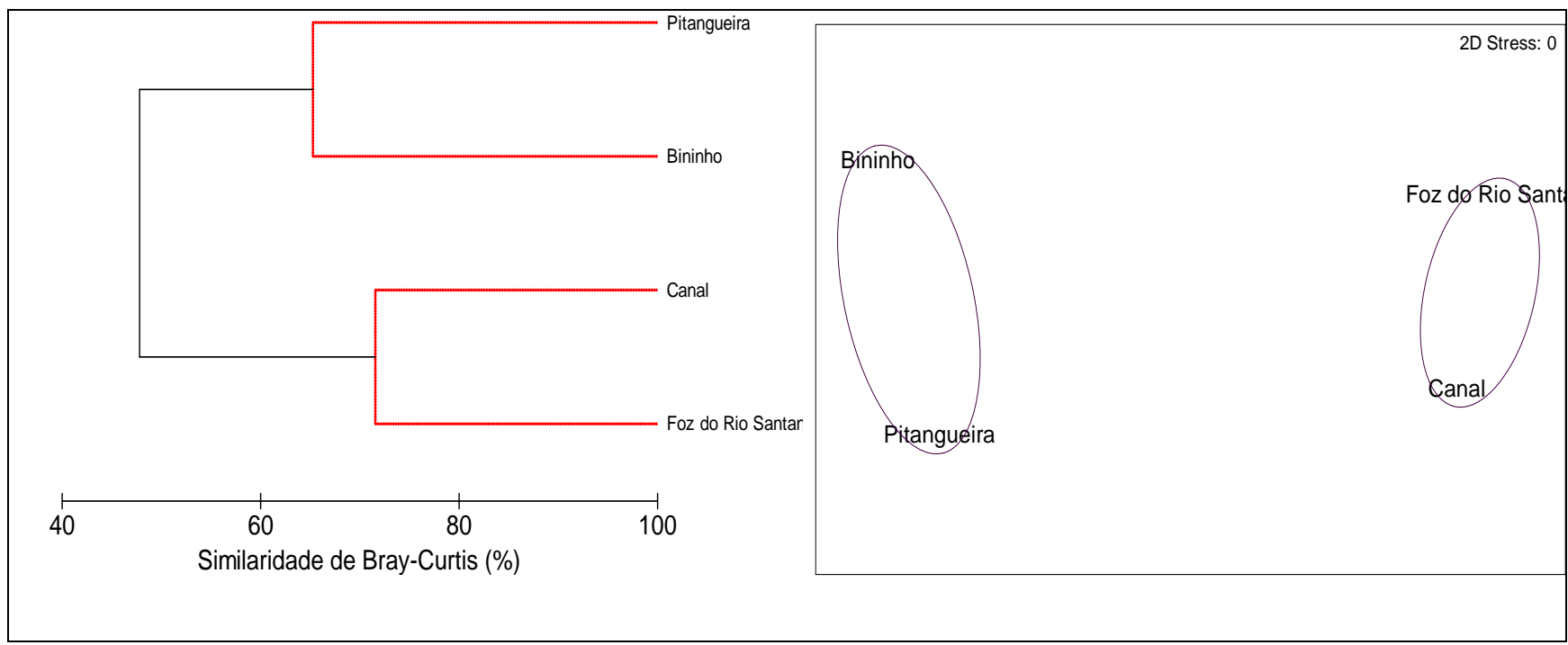

Figura 10 - Cluster e MDS utilizando como atributos a ocorrência e a abundância por ponto de coleta das espécies capturadas em Conceição da Barra, Espírito Santo

Mudanças espaciais com base na composição em espécies e suas abundâncias, não foram observadas entre as assembleias de peixes coletadas no Cais, foz do rio Mariricu, Transpetro e rio Mariricu, áreas amostradas no estuário de Barra Nova (Fig. 11). Em termos ictiofaunisticos estas quatro áreas apresentaram entre si, uma similaridade de $72 \%$. Mesmo não diferindo significativamente entre si, observou-se uma maior proximidade faunística entre as áreas Cais e rio Mariricu e entre foz do rio Mariricu e Transpetro.

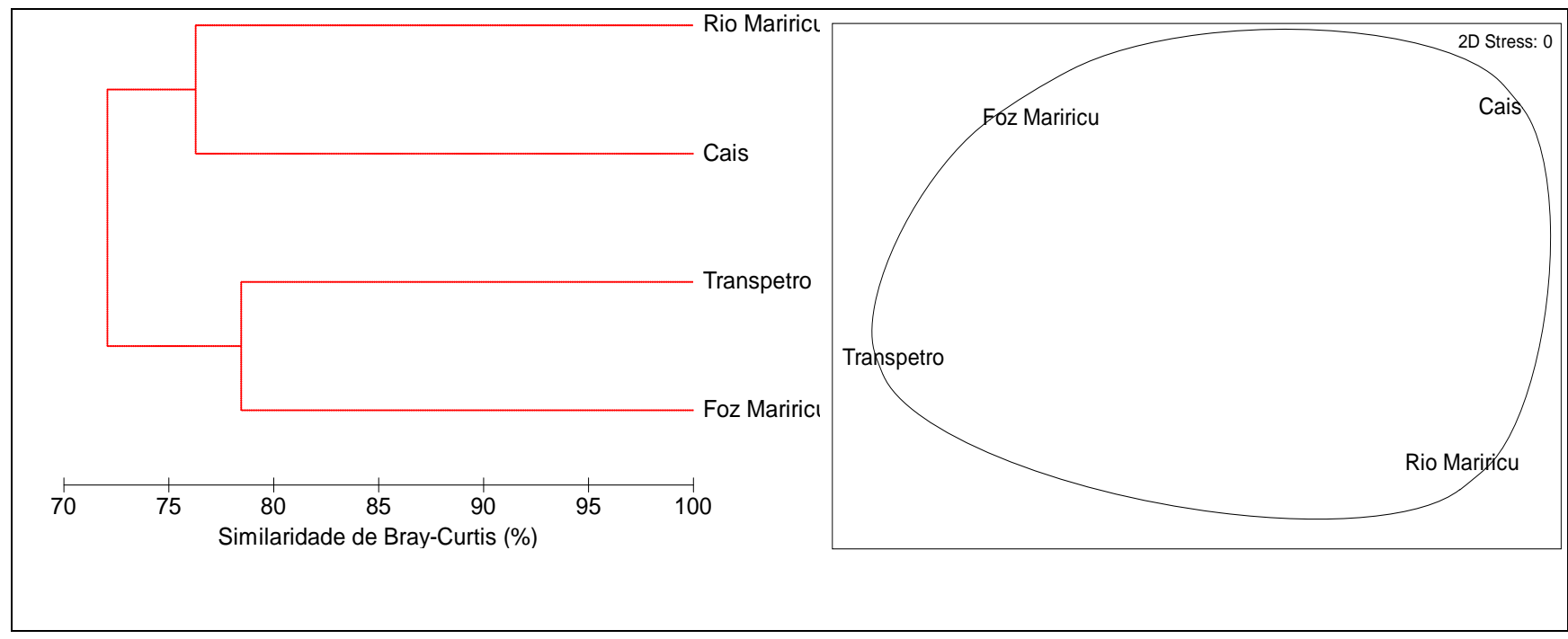

Figura 11 - Cluster e MDS utilizando como atributos a ocorrência e a abundância por ponto de coleta das espécies capturadas na Barra Nova, Espírito Santo.

\section{DISCUSSÃO}

A diversidade de espécies registrada nesse estudo é menor que os valores registrados em outros estudos da ictiofauna em estuários brasileiros (BARLETTA et al., 2003; BARLETTA et al., 2008; CHAGAS et al., 2006 e SCHWARZ et al., 2006). No entanto, são similares aos valores encontrados por Queiroz et al. (2006), para a ictiofauna demersal no Complexo Estuarino de Paranaguá (PR) e também similar aos estudos de Paiva et al. (2008) em estuário de águas rasas em Pernambuco.

Estudos apontam que o litoral do Espírito Santo está localizado em uma zona de transição entre ambientes estuarinos distintos, um ao norte e outro sul da costa brasileira (FLOETER et 
M. HOSTIM-SILVA et al. As assembleias de peixes dos estuários de conceição da barra e barra nova, espírito santo.

al., 2001; JOYEUX et al., 2001; FERREIRA et al., 2004; CHAGAS, 2005). Sendo assim, os estuários capixabas apesar de suas particularidades possuem características similares a outros estuários brasileiros, no entanto as semelhanças entre os estuários brasileiros limitam-se ao táxon família (CHAGAS, 2005).

As diferenças existentes podem ser atribuídas à dificuldade de comparar dados de riqueza entre diferentes estuários, uma vez que alguns fatores como a heterogeneidade de habitats, diferenças na composição físico-química do ambiente e os esforços de captura são variáveis (ARAÚJO et al., 1998). Importante considerar também o conhecimento e experiência no manejo dos apetrechos de pesca e definição das áreas de amostragem.

A presença de apenas três taxa da ictiofauna de água doce nos estuários norte do Espírito Santo parece ser uma característica de estuário tropical e temperado, sugerindo níveis acentuados de salinidade em estuário dominado por marés (VIEIRA; MUSICK, 1993; VILAR et al., 2010). A dominância da assembléia de peixes marinhos é outra característica geral em estuários tropical e temperado do Atlântico Oeste (KENNISH, 1986; VIEIRA; MUSICK, 1993). Esta dominância também encontrado nesse trabalho corroborando com dados de QUEIROZ et al. (2006) que destaca a ocorrência de grande número de espécies ocasionais com contribuição menor que $1 \%$ do total.

As famílias Sciaenidae e Gerreidae (ARAÚjO et al., 1998; SCHWARZ et al., 2006) e Gerreidae (ARAÚjO et al., 1998; BARLETTA et al., 2003 CHAGAS et al., 2006; PAIVA et al., 2008), abundantes nesse estudo, foram bem diversificadas em outros estuários. Nos estuários de Conceição da Barra e Barra Nova a representatividade das famílias se deve a abundância de indivíduos Stellifer sp. e Diapterus rhombeus.

A dominância de Sciaenidae era esperada, uma vez que regiões tropicais e subtropicais apresentam características estuarinas similares a outras regiões zoogeográficas no mundo, favorecendo a adaptação para as espécies dessa família (BLABER, 2002). Segundo Figueiredo E Menezes (1980), os ciaenideos são comuns em águas rasas com fundos de areia ou lama, coincidindo com o tipo de substrato observado nas áreas de coletas deste estudo.

Segundo Chaves; Otto (1998) D. rhombeus é uma espécie marinha migrante muito comum em ambientes estuarinos e de manguezais, embora apresente pequeno porte, a abundância de indivíduos pode ter grande importância na dinâmica biológica desses ambientes. Além disso, $D$. rhombeus é tolerante a baixas salinidades e podem ser dominantes em fase juvenil nos ambientes estuarinos mais interiores de mangue e áreas rasas de praia, principalmente estuários sob forte influência marinha. Já indivíduos adultos procuram áreas mais profundas para desova (FIGUEIREDO; MENESES, 1980; PAIVA et al. 2008; VILAR et al., 2010).

Fatores bióticos e abióticos podem interferir na composição e abundância de espécies nos estuários. Tem-se sugerido que os fatores abióticos sobrepõem os bióticos nessa determinação. Dentre os fatores abióticos, destacam-se a salinidade e temperatura (BLABER, 2000).

A alta abundância de indivíduos e diversidade de espécies de peixes foi observada no período compreendido entre os meses de novembro e abril (época em que foram observadas as maiores temperaturas no ambiente), exceto para o estuário de Conceição da Barra que apresentou um número grande de indivíduos coletados também no mês de junho. No entanto, no estuário de Conceição da Barra diferenças significativas só foram observadas entre as médias mensais do número de indivíduos, já no estuário de Barra Nova só as médias mensais da diversidade não foram significativamente diferentes.

Apesar da ausência de dados sobre os parâmetros hidrográficos, o agrupamento do Cluster para ocorrência e abundância mensal das espécies no estuário de Conceição da Barra tende apresentar uma resposta, principalmente, a variação na temperatura, com os meses de menor média mensal (junho a agosto) que não corresponderam ao período de menor média de precipitação. Embora transparência e temperatura sejam considerados fatores secundários à salinidade e profundidade na distribuição da ictiofauna, Marshall e Elliott (1998) no Estuário Humber na Inglaterra e Vilar et al. (2010) na Baía de Babitonga em Santa Catarina, relacionam a variação sazonal da temperatura, com processos no ciclo de vida (migração, reprodução e 
M. HOSTIM-SILVA et al. As assembleias de peixes dos estuários de conceição da barra e barra nova, espírito santo.

recrutamento), provavelmente responsáveis pela variação temporal na distribuição da ictiofauna nesses ambientes.

Louis et al. (1995) afirmam que os valores mais elevados de riqueza e diversidade são encontrados nas estações mais quentes do ano. O que vem a corroborar com resultados encontrados em estudos realizados por outros autores sobre ictiofauna demersal (QUEIROZ et al., 2006; GODEFROID et al., 2004; BRANCO; VERANI, 2006).

Diferenças espaciais só não foram significativas entre as médias da equitatividade e do número de indivíduos nos estuários de Conceição da Barra e Barra Nova, respectivamente. Diferenças significativas entre as assembleias de peixes dos pontos de coleta foram observadas no estuário de Conceição da Barra. O estuário de Conceição da Barra foi dividido em dois grupos definidos nas análises de agrupamento e ordenamento. Um compreende as áreas mais reservadas do estuário (canais de mangue) onde as florestas de mangue exercem grande influência sobre o meio, os pontos Bininho e Pitangueira. Um segundo grupo compreende as áreas mais abertas do estuário com alto fluxo de água (baixa influência das florestas de mangue), o Canal principal do rio e a Foz do Rio Santana.

A dominância de algumas espécies em alguns meses do ano, especialmente no final das estações seca e chuvosa podem indicar um processo de migração entre áreas, provavelmente associada à variação na salinidade, pelo incremento de água doce (BARLETTA et al., 2003; BARLETTA, et al., 2008). Aparentemente as áreas do Bininho e Pitangueira, mais isoladas pela geografia do estuário de Conceição da Barra, estão menos sujeitas às oscilações que as áreas do Canal e Foz do Rio Santana e por isso apresentam diferenças significativas.

Alguns estudos relatam que as florestas de mangue exercem grande influência sobre a ictiofauna em estuários (NAGELKERKEN et al., 2000), a qual produz elevada produção de detrito vegetal. Chaves e Vendel (2008) afirmam que o ponto inicial de obtenção de energia pelos peixes de estuário está no habito detritívoro da matéria vegetal por algumas espécies. Outros fatores que influenciam na abundância de peixes nos estuários é a utilização desses ambientes para reprodução e migração (BLABER, BLABER, 1980), seja nos estágios iniciais ou durante todo o ciclo de vida (LAFFAILLE et al., 2000).

A abundância média de duas espécies de bagres (Genidens genidens e Pseudauchenipterus nodosus) foi primordial na separação das áreas abertas (Canal e Foz do Rio Santana) em relação às áreas fechadas (Bininho e Pitangueira). Houve grande captura de Genidens genidens em ambas as áreas abertas no mês de dezembro, período de maiores temperatura. Os indivíduos coletados apresentavam elevado número de alevinos e ovos transportados na boca, demonstrando se tratar de uma importante área de recrutamento dessa espécie.

A instabilidade ambiental, principalmente causada pelo regime de chuvas pode induzir a movimentação da assembléia de peixes para áreas estuarinas mais estáveis. Contudo, algumas espécies, como Genidens genidens, mais tolerantes às oscilações de salinidade, podem permanecer em altas densidades de indivíduos juvenis para evitar a concentração de peixes nas áreas mais estáveis (BARLETTA, et al., 2003; BARLETTA et al., 2008). Segundo GOMES et al. (1999) G. genidens apresenta variação temporal associado ao período reprodutivo da espécie. A abundância de machos e fêmeas é baixa no período entre junho e setembro e com picos de abundância de fêmeas (dezembro e janeiro) e de machos (fevereiro e março) durante o verão, - que explica a abundância da espécie encontrada no período chuvoso, devido ao grande número de fêmeas com ovócitos. Já Queiroz et al. (2006) sugere que G. genidens tende a estar associado também à ambientes com menores profundidades.

O Pseudauchenipterus nodosus foi largamente capturada entre os meses de novembro a dezembro, sendo o mês de dezembro época de reprodução dessa espécie, peixe de habito detritívoro é largamente encontrado próximo a áreas de despejo de águas residuais de cidades (LE BAIL et al. 2000), ressaltamos que as áreas abertas estudadas estão localizadas próximo ao cais da cidade. Tem preferência por grandes rios e águas ligeiramente salobras, mais próxima de doce (KENNY, 1995; BOUJARD et al. 1997). 
M. HOSTIM-SILVA et al. As assembleias de peixes dos estuários de conceição da barra e barra nova, espírito santo.

Mudanças espaciais com base na composição em espécies e suas abundâncias, não foram observadas entre as assembleias de peixes coletadas nos pontos estudados para o estuário de Barra Nova. Isso pode estar relacionado à similaridade existente entre os quatros ambientes estudados. No entanto observou-se uma maior proximidade faunística entre as áreas Cais e rio Mariricu e entre foz do rio Mariricu e Transpetro.

\section{CONCLUSÃO}

De um modo geral, as ictiofaunas dos dois estuários apresentaram pequenas diferenças entre si. O número de exemplares e espécies amostrados foi semelhante, foi baixa a ocorrência de espécies de água doce e a diversidade por família esteve próxima. O predomínio em número e peso foi exercido pelas mesmas espécies, com um grande número de espécies contribuindo cada uma com menos de $1 \%$ da captura de cada área. Mesmo as espécies exclusivas, por estarem presentes, na maioria dos casos, em pequena quantidade, parecem não definir preferência ambiental. Por outro lado, diferenças nos padrões temporais e espaciais na estrutura e composição das assembleias de peixes foram evidentes entre as duas áreas amostrais.

\section{REFERÊNCIAS BIBLIOGRÁFICAS}

ARAÚJO, F. G. Adaptação do índice de integridade biótica usando a comunidade de peixes para o rio Paraíba do Sul. Revista Brasileira de Biologia, v. 58, n. 4, p. 547-558, 1998.

ATTRILL, M. L.; RUNDLE, S. D. Ecotone or Ecocline: ecological boundaries in estuaries.

Estuarine, Coastal and Shelf Science,v. 5, p. 929-936, 2002.

BARLETTA, M.; CORRÊA, M. F. M. Guia para identificação de peixes da costa do Brasil. Curitiba, Ed. da UFPR, 1992, p.131.

BARLETTA, M.; BARLETTA-BERGAN, A.; SAINT-PAUL, U.; HUBOLD, G. Seasonal changes in density, biomass and diversity of estuarine fishes in tidal mangrove creeks of the lower Caeté Estuary (northern Brazilian coast, east Amazon). Marine Ecology Progress Series, v. 256, p. 217-228, 2003.

BARLETTA, M.; AMARAL, C. M.; CORRÊA, M. F. M.; GUEBERT, F.; DANTAS, D. V.; LORENZI, L.; SAINT-PAUL, U. Factors affecting seasonal variations in demersal fish assemblages at an ecocline in a tropical-subtropical estuary. Journal of Fish Biology, v. 73, p. 1314-1336, 2008.

BLABER, S. J. M.; BLABER, T. G. Factors afecting the distribution of juvenile estuarine and inshore fish. Journal of Fish Biology v. 17, p. 143-162, 1980.

BLABER, S. J. M. 'Fish in hot water': the challenges facing fish and fisheries research in tropical estuaries. Journal of Fish Biology v. 61, (Supplement A),p. 1-20, 2002.

BOUJARD, T.; PASCAL, M.; MEUNIER, F. J.; LE BAIL P.-Y. Poissons de Guyane. Guide écologique do Approuague te de a réserve des Nouragues. Instituto National da Recherche

Agronomique, Paris, p.219,1997.

BRANCO, J. O.; VERANI, J. R. Análise quali-quantitativa de ictiofauna acompanhante na pesca do camarão sete-barbas, na Armação do Itapocory, Penha, Santa Catarina. Revista Brasileira de Zoologia, v. 23, n. 2, p. 381-391, 2006.

CARMOUZE, J. P. O metabolismo dos ecossistemas aquáticos. São Paulo: Ed.Edgard Blücher/Sbl, 1994.

CHAGAS, L. P. Aspectos zoogeograficos da ictiofauna estuarina no Atlântico oeste. 2005. Monografia (Bacharelado em Oceanografia), Universidade Federal do Espírito Santo, Vitória.

CHAGAS, L. P., JOYEUX, J. C; FONSECA, F. R. Small scale spatial changes in estuarine fish subtidal assemblage in tropical Brazil. Journal of Marine Biology Association of the United Kingdon, v.86, p. 861-875, 2006.

CHAVES, P. T. C.; OTTO, G. Aspectos Biológicos de Diapterus rhombeus (Cuvier) (Teleostei, Gerreidae) na Baía de Guaratuba, Paraná, Brasil. Revista brasileira de Zoologia, v. 15, n. 2, p. 289-295, 1998. 
M. HOSTIM-SILVA et al. As assembleias de peixes dos estuários de conceição da barra e barra nova, espírito santo.

CHAVES, P. T. C, ; VENDEL, A. L. Análise comparativa da alimentação de peixes (Teleostei) entre ambientes de marisma e de manguezal num estuário do sul do Brasil (Baía de Guaratuba, Paraná). Revista brasileira de Zoologia. v. 25, n. 1, p. 10-15, 2008.

CLARKE, K. R.; WARWICK, R. W. Change in marine communities: an aproach to statistical analysis and interpretation. [S.I.]: Plymouth Marine Laboratory 1994, p.859.

COSER, L. M., PEREIRA, B. B.; JOYEUX, J-C. Descrição da comunidade ictioplanctônica e sua distribuição espacial no estuário dos rios Piraquê-Açu e Piraquê-Mirim, Aracruz, ES, Brasil.

Interciencia, v. 32, n. 4, p. 233-241, 2007.

CYRUS, D. P.; BLABER, S. J. M. Turbidity and salinity in a tropical Northern Autralian estuary and their influence on fish distribution. Estuarine, Coastal and Shelf Science, v. 35, p. 545$563,1992$.

DAY, J. W. JR, HALL, C. A. S., KEMP, W. M.; YÁNEZ-ARANCIBIA, A. Estuarine ecology. New York. John Wiley; Sons, 1989, p. 558.

FERREIRA, C. E. L., FLOETER, S. R., GASPARINI, J. L., FERREIRA, B. P.; JOYEUX, J.-C. 2004. Trophic structure patterns of Brazilian reef fishes: a latitudinal comparison. Journal of Biogeography n. 31, p. 1093-1106, 2004.

Figueiredo, J. L.; MeneZes, N. Manual de Peixes Marinhos do Sudeste do Brasil - II. Teleostei (1). Museu de Zoologia, Universidade de São Paulo, São Paulo, 1978, p.110.

Figueiredo, J. L.; MENEZeS, N. Manual de Peixes Marinhos do Sudeste do Brasil - III. Teleostei (2). Museu de Zoologia, Universidade de São Paulo, São Paulo, 1980, p.90.

FIgueiredo, J. L.; MENeZeS, N. Manual de Peixes Marinhos do Sudeste do Brasil - VI. Teleostei (5). Museu de Zoologia, Universidade de São Paulo, São Paulo, 2000 p.116.

Floeter, S. R., GuimarÃES, R. Z. P., ROCHA, L. A., FERREIRA, C. E. L., RANGEL, C. A. ; GASPARINI, J. L. Geographic variation in reef-fish assemblages along the Brazilian coast. Global Ecology and Biogeography, v. 10, p. 423-433, 2001.

GODEFROID, R. S.; SPACH, H. L.; SANTOS, C.; MACLAREN, G.; SCHWARZ-JUNIOR, R. Mudanças temporais na abundância e diversidade da fauna de peixes do infralitoral raso de uma praia, sul do Brasil. Iheringia, Série Zoologia, v. 94, n. 1, p.95-104, 2004.

GOMES, I. D., ARAÚJO, F. G., AZEVEDO, M. C. C.; PESSANHA, A. L. M. Biologia reprodutiva dos bagres marinhos Genidens genidens (Valenciennes) e Cathorops spixii (Agassiz) (Siluriformes, Ariidae), na baía de Sepetiba, Rio de Janeiro, Brasil. Revista brasileira de Zoologia v. 16, p.171-180, 1999.

HYNDES, G. A., PLATELL, M. E., POTTER, I. C.; LENANTON, R. C. J. Does the composition of the demersal fish assemblages in temperate coastal waters change with depth and undergo consistent seasonal changes? Mar. Biol., v. 134, p. 335 - 352, 1999.

JAUREGUIZAR, A. J.; MENNI, R.; BREMEC, C.; MIANZAN, H.; LASTA, C. Fish assemblage and environmental patterns in the Río de La Plata estuary. Estuarine, Coastal and Shelf Science, v. 56, p. 921-933, 2003.

JAUREGUIZAR, A. J.; MENNI, R.; GUERRERO, R.; LASTA, C. Environmental factors struturing fish communities of the Río de La Plata estuary. Fisheries Research, v. 66, p. 195-211, 2004.

JOYEUX, J.-C., FLOETER, S. R., FERREIRA, C. E. L.; GASPARINI, J. L. Biogeography of tropical reef fish: the South Atlantic puzzle. Journal of Biogeography, v. 28, p. 831-841, 2001.

JOYEUX, J.-C., PEREIRA, B.B.; ALMEIDA, H.G. The Floodtide ichthyoplanktonic community at the entrance into a Brazilian tropical estuary. Journal of Plankton Research, v. 26, p. 12771287, 2004.

KENNISH, M. J. Ecology of estuaries (1). CRC Press, Boston, 1986, p 253.

KENNY, J. S. Views from the bridge: a memoir onde the freshwater fishes of Trinidad. Julian S. Kenny, Maracas, St. Joseph, Trinitat e Tobago, 1995, 98 p. 
M. HOSTIM-SILVA et al. As assembleias de peixes dos estuários de conceição da barra e barra nova, espírito santo.

KUPSCHUS, S.; TREMAIN, D. Associations between fish assemblages and environmental factors in nearshore habitats of a subtropical estuary. Journal of Fish Biology, v. 58, p. 1383-1403, 2001.

LE BAIL, P. Y; KEITH, P.; PLANQUETTE, P. Atlas des poissons d'eau douce de Guyane. Tomo II. Paris: Muséum National d'Histoire Naturelle. 2000.

LONERAGAN, N. R.; POTTER, I. C.; LENANTON, R. C. J.; CAPUTI, N. Influence of environmental variables on the fish fauna of the deeper waters of a large Australian estuary. Marine Biology, v. 94, p. 631-641, 1987.

LONERAGAN, N. R.; POTTER, I. C.; LENANTON, R. C. J. Influence of site, season and year on contributions made by marine, estuarine, diadromous and freshwater species to the fish fauna of a temperate Australian estuary. Marine Biology, [S.I.], v. 103, p. 575-586, 1989.

LOUIS, M.; BOUCHON, C.; BOUCHON-NAVARRO, Y. Spatial and temporal variations of mangrove fish assemblages in Martinique (French West Indies). Hydrobiologia, v. 295, p.275284, 1995.

MACIEIRA, R. M. Aspectos da ictiofauna do sistema estuarino dos rios Piraquê-açu e Piraquê-mirim, E.S., 2005. Monografia, Oceanografia, Universidade Federal do Espírito Santo, p.50.

MAES, J.; VAN DAMME, P. A.; TAILLIEU, A.; OLLIVIER, F. Fish communities along na oxygenpoor salinity gradient (Zeeschelde Estuary, Belgium). Journal of Fish Biology, [S.I.], v. 52, p. 534-546, 1998.

MARSHALL, S.; ELLIOTT, M. Environmental influences on the fish assemblage of the Humber estuary. Estuarine, Coastal and Shelf Science, v. 46, p. 175-184, 1998.

Menezes, N.; FIgueiredo, J. L. Manual de Peixes Marinhos do Sudeste do Brasil - IV. Teleostei (3). Museu de Zoologia, Universidade de São Paulo, São Paulo, 1980, p.96.

Menezes, N.; FIgUeiredo, J. L. Manual de Peixes Marinhos do Sudeste do Brasil - V. Teleostei (4). Museu de Zoologia, Universidade de São Paulo, São Paulo, 1985, p.105.

NAGELKERKEN, I.; VAN DER VELD, G.; GORISSEN, M.W.; MEIJER, G.J.; VAN'THOF, T.; DEN HARTOG, C. Importance of mangroves, seagrass beds and the shallow coral reef as a nursery for importance coral reef fish, using a visual census technique. Estuarine, Coastal and Shelf Science, n. 51, p. 31-44, 2000.

PAIVA, A. C. G.; CHAVES, P. T. C.; ARAÚJO, M. E. Estrutura e Organização Trófica da Ictiofauna de Águas Rasas em um Estuário Tropical. Revista brasileira de Zoologia, v. 25, n. 4,p. 647$661,2008$.

PATTERSON, A. W.; WHITFIELD, A. K. Do the shallow-water habitats function as refugia for juveniles fishes? Estuarine, Coastal and Shelf Science, v.51,p.359-364, 2000.

QUEIROZ, G. L. M. N.; SPACH, H. L.; SOBOLEWSKI-MORELOS, M.; SANTOS, L. O.; SCHWARZ JR, R. Caracterização da Ictiofauna demersal de duas áreas do complexo estuarino de Paranaguá, Paraná, Brasil. BIOCIÊNCIAS, v. 14, n. 2, p. 112-124, 2006.

RESENDE, F. Análise da variabilidade da utilização de informações a respeito da comunidade de peixes e de variáveis ambientais no monitoramento da qualidade da água em canais de mangue do estuário da baía de Vitória. 2005. Dissertação de Mestrado, Engenharia Ambiental, Universidade Federal do Espírito Santo, 134p.

SCHWARZ JR, R.; FRANCO, A. C. N. P.; SPACH, H. L.; SARPEDONTI, V.; PICHLER, H. A.; QUEIROZ, G. M. L. N. Composição e Estrutura da Ictiofauna Demersal na Baía dos Pinheiros, Paraná. Braz. J. Aquat. Sci. Technol., v.10, n. 1, p. 27-39, 2006.

SILVA, M. A. B. da; BERNINII, E.; CARMO, T. M. S.Características estruturais de bosques de mangue do estuário do rio São Mateus, ES, Brasil. Acta bot. bras.,v. 19, n. 3, p. 465-471, 2005. 
M. HOSTIM-SILVA et al. As assembleias de peixes dos estuários de conceição da barra e barra nova, espírito santo.

SOKAL, R. R.; ROHLF, F. J. Biometry the principles and practice of statistics in biological research. W. H. Freeman and Co., 3d. ed. San Francisco, 1995, p. 887.

VIEIRA, J. P.; MUSICK, J. A. Latitudinal patterns in diversity of fishes in warm-temperate and tropical estuarine waters of the western Atlantic. Atlântica, v. 15, p. 115-133, 1993.

VILAR, C. C.; SPACH, H. L.; JOYEUX, J. C. Spatial and temporal changes in the fish assemblage of a subtropical estuary in Brazil: environmental effects. Journal of the Marine Biological Association of the United Kingdom. p. 1-14. 2010

WHITFIELD, A. K. Ichthyofaunal assemblages in estuaries: a South African case study. Reviews in Fish Biology and Fisheries. v. 9, p. 151-186, 1999. 